\title{
Attractors in Dark Energy Models with Born-Infeld Scalar Field
}

\author{
Ronaldo Carlotto Batista, Luís Raul Weber Abramo, and Thiago dos Santos Pereira \\ Instituto de Física, Universidade de São Paulo, CP 66318, 05315-970, São Paulo, Brazil
}

(Received on 17 October, 2005)

\begin{abstract}
We derive, in the large scale limit, analytical solutions for a dark energy model described by the Born-Infeld field plus a perfect fluid, both for homogeneous background and first order perturbations. These analytical results are compared with numerical solutions in a model with radiation, pressureless matter and the field. We investigate the non-adiabatic perturbed pressure associated with this attractor and whether it corresponds to isocurvature contribution.
\end{abstract}

\section{INTRODUCTION}

Recent observations [1] indicate that expansion of the universe is accelerated. Models with cosmological constant $\Lambda$ and canonical scalar fields [2] have been proposed to explain this acceleration. Another class of scalar field theory is the Born-Infeld theory [3]:

$$
\mathcal{L}=\sqrt{-g} V(\varphi) \sqrt{1+\partial^{\mu} \varphi \partial_{\mu} \varphi} .
$$

We use a inverse power-law potential $V(\varphi)=M^{4+\alpha} \varphi^{-\alpha}$, where, for dark energy models, we must have $0 \leq \alpha<2$ [4]. The equation of motion of such field is:

$$
\nabla^{\mu} \partial_{\mu} \varphi+\frac{\nabla_{\mu} \partial_{\nu} \varphi}{1+\partial_{\mu} \varphi \partial^{\mu} \varphi} \partial^{\mu} \varphi \partial^{v} \varphi+\ln (V)_{, \varphi}=0,
$$

which, at homogeneous level becomes

$$
\frac{\ddot{\varphi}}{1-\dot{\varphi}^{2}}+3 H \dot{\varphi}+\ln (V)_{, \varphi}=0 .
$$

In this case, the density and pressure are:

$$
\rho_{\varphi}=\frac{V(\varphi)}{\sqrt{1-\dot{\varphi}^{2}}}, p_{\varphi}=-V(\varphi) \sqrt{1-\dot{\varphi}^{2}},
$$

and $\omega_{\varphi}=p_{\varphi} / \rho_{\varphi}$.

The background evolution is given by the Friedmann equation, with $8 \pi G=1$,

$$
H^{2}=\left(\frac{\dot{a}}{a}\right)^{2}=\frac{1}{3}\left(\rho_{r}+\rho_{m}+\rho_{\varphi}\right),
$$

the equation (3) plus the equations of continuity for radiation and matter.

\section{ATTRACTORS}

The equation (3) may assume the solution $\ddot{\varphi}=0$. When a perfect fluid with equation of state $\omega_{f}=\gamma_{f}-1$ dominates the background evolution, we have $H=\frac{2}{3 \gamma_{f} t}$ and the field grows linearly in time [4]:

$$
\varphi=A t
$$

where $A=\sqrt{\frac{\alpha \gamma_{f}}{2}}$ for $\alpha<2 / \gamma_{f}$, and $A=1$ for $\alpha>2 / \gamma_{f}$.

We evaluate first order scalar perturbations in the newtonian-longitudinal gauge [5], with no anisotropic stress:

$$
d s^{2}=-(1+2 \Phi) d t^{2}+a^{2}(t)(1-2 \Phi) d \vec{x}^{2} .
$$

In this case, the first order perturbations of the field obey the equation:

$$
\begin{gathered}
\frac{\delta \ddot{\varphi}}{1-\dot{\varphi}^{2}}+\left[3 H+\frac{2 \ddot{\varphi} \dot{\varphi}}{\left(1-\dot{\varphi}^{2}\right)^{2}}\right] \delta \dot{\varphi}+\left[\frac{k^{2}}{a^{2}}+\ln (V(\varphi))_{, \varphi \varphi}\right] \delta \varphi \\
=2\left[3 H \dot{\varphi}+\frac{\ddot{\varphi}}{\left(1-\dot{\varphi}^{2}\right)^{2}}\right] \Phi+4 \dot{\varphi} \dot{\Phi}
\end{gathered}
$$

In the large scale limit $k / a H \ll 1$, assuming $\dot{\Phi}=0, H=$ $\frac{2}{3 \gamma_{f} t}$, and the background solution (6), the field perturbation has the following attractor solution:

$$
\delta \varphi=A \Phi t+c_{1} t^{-1}
$$

where $c_{1}$ is a constant. Neglecting the decaying mode, we have

$$
\frac{\delta \varphi}{\varphi}=\Phi
$$

In this case, the field evolves as a perfect fluid with constant equation of state, given by $\omega_{\varphi}=A^{2}-1$.

When the attractor regime is reached at matter domination, we have $\gamma_{f}=1$, then always $\alpha<2 / \gamma_{f}$ and $A=\sqrt{\frac{\alpha \gamma_{f}}{2}}$. But, when the attractor regime is reached at radiation domination, we have $\gamma_{f}=4 / 3$, then we may have $\alpha<2 / \gamma_{f}=1.5$, thus $A=\sqrt{\frac{\alpha \gamma_{f}}{2}}$, or $\alpha>2 / \gamma_{f}=1.5$ and $A=1$. In the latter case, the field perturbations can grow indefinitely when the transition between radiation domination and matter domination is going on. This behaviour is strongly dependent on the initial conditions for the field perturbations. Hence, we call models with $\alpha<1.5$ "stable" and with $\alpha>1.5$ "unstable".

\section{ISOCURVATURE PERTURBATION}

We want to investigate, in the large scale limit, whether the Born-Infeld field generates isocurvature perturbations. The 


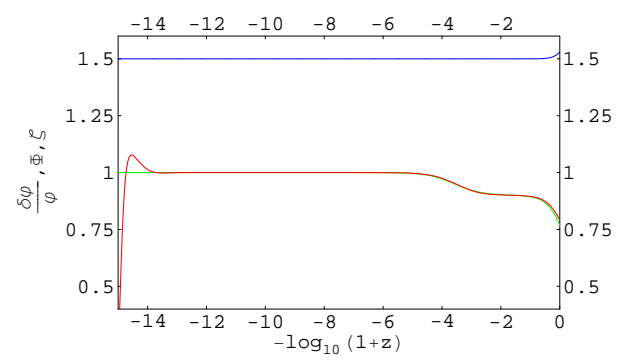

FIG. 1: for $\alpha=1$, the evolution of $\delta \varphi / \varphi$ (red line), $\Phi$ (green line) and $\zeta$ (blue line). The attractor solutions are well verified, even during the transition $\left(z \approx 10^{4}\right)$. Note that the $\zeta$ function is constant almost all the time, only when the field starts to dominate this function starts do vary, this shows that the field induces some nonadiabatic pressure.

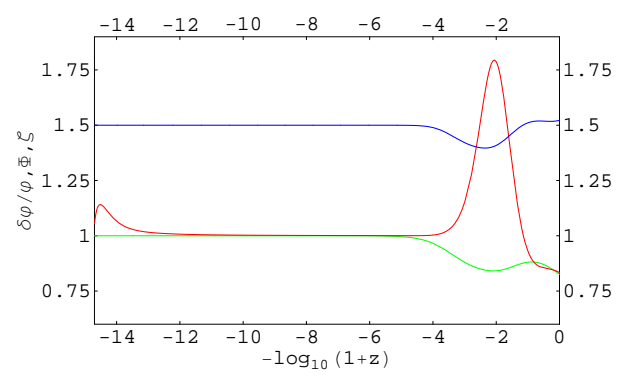

FIG. 2: for $\alpha=1.51$, the evolution of $\delta \varphi / \varphi$ (red line), $\Phi$ (green line) and $\zeta$ (blue line). The attractor solutions are well verified at radiation dominated era. During the transition $\left(z \approx 10^{4}\right)$, the field perturbation escapes from the attractor and generates isocurvature modes, what can be seen from the variation of the $\zeta$ function at this period. Here we have chosen initial conditions for the field perturbations such that $\delta \varphi$ roughly reaches the modified attractor at matter dominated era.

function [6]

$$
\zeta=\frac{2}{3} \frac{\Phi^{\prime}+\Phi}{\left(1+\omega_{t o t}\right)}+\Phi
$$

where the prime denotes the derivative with respect to $N=$ $\ln \left(a / a_{0}\right)$, has the following equation of motion $[5,6]$

$$
\zeta^{\prime}=\frac{\delta p_{\text {nad }}}{\left(1+\omega_{t o t}\right) \rho_{t o t}},
$$

where $\delta p_{\text {nad }}$ is the total nonadiabatic pressure. From (12), we see that $\zeta$ can only vary in large scales if the nonadiabatic pressure is nonnegligible.

To verify if the Born-Infeld field generates isocurvature modes we set adiabatic conditions between matter and radiation, $\delta_{m_{i}}=0.75 \delta_{r_{i}}$, and evaluate the functions $\delta \varphi / \varphi, \Phi$ and $\zeta$ for $\alpha=1$ (stable case - Figure 1) and $\alpha=1.51$ (unstable case - Figure 2) .

To illustrate the dependence of the evolution of the field perturbations on its initial conditions and how this can generate isocurvature modes, we show in Figure 3, for three different sets of initial conditions, the evolution of $\zeta$ function, for three different values of $\alpha$.

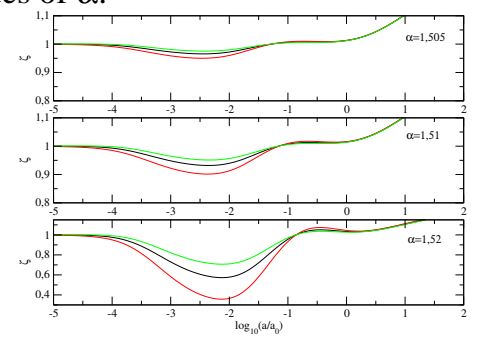

FIG. 3: evolution of the $\zeta$ function, for three different sets of initial conditions for the field perturbations. Note that the variation of $\zeta$ grows with $\alpha$.

\section{CONCLUSIONS}

For dark energy models, the isocurvature modes tend to be very small. This occurs because to approach $\omega_{\varphi}=-1$ today, we need $\alpha$ near zero, what decreases the isocurvature modes. On the other hand, since we have $\alpha>1.5$, the isocurvature modes can be very significant. But, in this case, we do not have good models for dark energy, once we have, in the matter dominated era, $\omega_{\varphi}=-0.25$ at least, what does not allow values of $\omega_{\varphi}$ close to -1 today. For this case, we have a model closer to a dark matter model.

\section{Acknowledgments}

This work was supported by CNPq and FAPESP.
[1] A. Riess et al, Astron. J. 116, 1009 (1998); C. Bennett et al., Astrophys. J. Suppl. 148, 1 (2003)

[2] B. Ratra and P. J. E. Peebles, Phys. Rev. D 37, 3406 (1988); C. Wetterich, Nucl. Phys. B302, 645 (1988).

[3] T. Padmanabhan, Phys. Rev.D 66, 021301 (2002); J. Bagla, H. Jassal, and T. Padmanabhan Phys. Rev. D 67, 063504 (2003); L. R. Abramo, F. Finelli and Thiago S. Pereira, Phys. Rev. D 70,
063517 (2004).

[4] L. R. Abramo and F. Finelli, Phys. Lett. B575, 165 (2003).

[5] V. F. Mukhanov, H. A. Feldman, and R. H. Brandenberger, Phys. Rept. 215, 203 (1992).

[6] J. M. Bardeen, P. J. Steinhardt, and M. S. Turner, Phys. Rev. D28, 679 (1983). 